\title{
Identification of breast cancer recurrence risk factors based on functional pathways in tumor and normal tissues
}

\author{
Xiujie Chen ${ }^{1, *}$, Lei Liu, ${ }^{1, *}$, Yunfeng Wang ${ }^{1, *}$, Bo Liu ${ }^{1, *}$, Diheng Zeng ${ }^{1}$, Qing Jin ${ }^{1}$, \\ Mengjian Li ${ }^{1}$, Denan Zhang ${ }^{1}$, Qiuqi Liu ${ }^{1}$, Hongbo Xie ${ }^{1}$ \\ ${ }^{1}$ College of Bioinformatics Science and Technology, Harbin Medical University, Harbin, Heilongjiang, 150081, PR China \\ "These authors contributed equally to this work
}

Correspondence to: Xiujie Chen, email: chenxiujie@ems.hrbmu.edu.cn

Keywords: breast cancer, normal tissue, tumor tissue, recurrence, risk factors

Received: September 30, 2015

Accepted: July 26, 2016

Published: August 23, 2016

\section{ABSTRACT}

The recurrence of breast cancer (BC) is a serious therapeutic problem, and the risk factors for recurrence urgently need to be identified. In this study, we examined the functional pathways in tumor and normal tissues to more comprehensively identify biomarkers for the risk of BC recurrence. We collected tumor and normal tissue gene expression profiles of recurrent BC patients and non-recurrent BC patients from the TCGA database.We derived an expression interval (mean $\pm 1.96 S D$ ) based on non-recurrent patients rather than a single value, such as a mean or median. If the expression of a gene was significantly different from its normal expression interval, it was considered a differentially expressed gene. Eight pathways that significantly distinguished recurrent and non-recurrent BC patients were obtained based on $65 \%$ accuracy, and these pathways were all associated with the immune response and sensitivity to drugs. The genes in these eight pathways were also used to analyze survival, and the significance level reached 0.003 in an independent dataset $(p=0.02$ in tumor and $p=0.03$ in normal tissue). Our results reveal that the integration of tumor and normal tissue functional analyses can comprehensively enhance the understanding of BC prognosis.

\section{INTRODUCTION}

Breast cancer $(\mathrm{BC})$ is a highly heterogeneous disease with different clinical manifestations. Although some patients can benefit from adjuvant systemic therapies, a substantial proportion of $\mathrm{BC}$ patients have poor therapeutic outcomes [1]. After chemotherapy, many patients develop adverse consequences of heavy metal poisoning, which decrease the success rate of therapy [2], and how to identify patients suitable for chemotherapy remains a challenge in the field. Many clinical and pathological factors are currently employed to predict the prognosis of patients, such as the Gallen guidelines [3], the National Institutes of Health guidelines [4], the Nottingham Prognostic Index guidelines [5] and the computer program Adjuvant! [6]. However, most of these prognosis factors rely on changes in the status of the patient's tumor, such as changes in the tumor diameter, axillary lymph node status, histologic grade, estrogen receptor (ER) status and progesterone receptor
(PR) status [7]. Several other cancer studies have found that precancerous tissue dysfunctions are associated with cancer prognosis, and these abnormal functions are often related to protective antitumor immunity [8]. In addition, the early identification of precancerous normal tissue damage may provide better prognosis biomarkers $[9,10]$. To this end, Ye M and Herszényi L considered the difference and uniformity between the precancerous and cancer statuses.Activated leukocyte cell adhesion molecule (ALCAM) has been identified as a novel potential molecular marker of gastric cancer. Ye M analyzed the serum soluble ALCAM (sALCAM) in large numbers of patients with gastric cancer, patients with precancerous lesions, and controls. The expression of ALCAM mRNA in different disease status was significantly different [11]. Herszényi L found that proteolytic enzymes play a major role not only in colorectal cancer (CRC) invasion and metastasis but also in the malignant transformation of precancerous lesions into cancer [12]. This finding indicates that the precancerous and cancer statuses may 
share common mechanisms that may influence prognosis. these findings show that precancerous and cancer status have not only differences in expression level but also uniformity in predicting prognosis Therefore, only considering the characteristics of cancer and ignoring precancerous characteristics is insufficient to predict prognosis. However, the studies combining these two statuses at the functional level to systematically assess cancer prognosis are rare. Therefore, we herein utilized both tumor and normal tissue from recurrent $\mathrm{BC}$ patients and from non-recurrent $\mathrm{BC}$ patients, identificated differentially expressed genes of precancerous normal tissue and tumor tissue between two patient groups and the biological processes affected by these genes. Finally, the utility of these genes to predict prognosis was evaluated.

Because breast cancer is highly heterogeneous, even in the patients with the same phenotype (such as good or poor outcome), these tumors also significantly exist differences at the molecular level. Due to this sample heterogeneity, genes that are only specifically expressed in small number groups are often not identified using traditional statistical methods, such as the $t$-test, Mann-Whitney test or the Significance Analysis of Microarrays (SAM) approach [13]. To identify DEGs between groups and genes that are specifically expressed in small number groups, we propose a new algorithm that uses the range of the expression levels in non-recurrent $\mathrm{BC}$ patients as a reference instead of comparing the mean expression levels between groups. Randomization was used to identify specific genes that were only altered in subgroups and reflect individual recurrence risk.

Thus, we used the randomization method to identify genes that express significantly beyond the normal interval.. We then identified pathways associated with recurrence risk in both normal and tumor tissues. In these pathways, patients with diverse prognosis present different functional level. We finally identified eight riskassociated pathways based on a transcriptional profile analysis that were efficiently able to predict $\mathrm{BC}$ patients with different prognosis outcomes. The risk-associated genes were differentially expressed genes in the riskassociated pathways. Calcium signaling, Gap junction, MAPK signaling and Jak-STAT signaling were obtained from tumor status, and explained the prognosis related functions in tumor therapeutic status.Tuberculosis, Salmonella infection, small cell lung cancer and influenza A were obtained in normal status study and showed the relationship between intrinsic genetic background and prognosis. Kaplan-Meier (K-M) survival curves indicated that patients without changes in risk-associated genes survived significantly longer than patients exhibiting changes. These findings demonstrate that the eight riskassociated pathways and genes can efficiently distinguish patients with good prognosis and patients with poor prognosis to predict recurrence risk. Our results can be used to objectively evaluate the need for further chemotherapy.

\section{RESULTS}

\section{Tumor tissue study}

\section{Differentially expressed gene analysis}

For each gene we calculated the value sum beyond normal interval based on good group, and obtained its probability distribution. The number of genes with a $p$ value less than 0.05 is 3852 genes. After FDR correction, we obtained 3254 genes, including 1923 up regulated genes and 1331 down regulated genes. (Supplementary Table S1).

\section{Comparing with the traditional method}

We used the $\mathrm{R}$ package of the Limma method to analyze the expression profile of tumor tissue and then compared this profile with our results. Specifically, the Limma algorithm returned 2684 differentially expressed genes, 2097 of which were also identified using our method. The normal tissue profiles were also analyzed, and the Limma algorithm identified 4565 differentially expressed genes, 3875 of which were also identified by our method.

However, we found that some significant differential expressed genes identified by Limma, i.e., DCAF17 and C7orf46, did not remain differentially expressed after random perturbation, as shown in Figure 1. These genes were considered as significantly expressed due to the mean difference between the two groups (recurrent and nonrecurrent) of data. However, according to the expression levels distribution, we found that these genes still fluctuate in the normal range, even if they have a different mean value with the control group (non-recurrent).

ANK2 and DCAF17 were extracted in tumor tissue of patients with different prognosis.After a randomization process, ANK2 was identified as differentially expressed $(P=0.0012)$, but this gene was not identified by the Limma algorithm $(P=0.07)$. Viewing from the expression value distribution, in spite of similar mean values between two groups, some samples in the poor prognosis group showed significantly higher expression level than the normal range, which shows that ANK2 may be involved in personalized relapse mechanism. For gene DCAF17, it was considered to be significant differentially expressed genes $(P=0.002)$ by the Limma algorithm, but was not significant after the process of randomization $(P=0.07)$. Although DCAF17 has different mean values between the two groups, it still fluctuate within the normal range. Similar results were obtained in normal tissue study, such as C7orf46 and CTHRC1. In conclusion, for some specific genes that are differentially expressed in small groups, traditional methods cannot identify them although there are differences between the groups at the mean level. Moreover, genes exhibiting a significant difference in their 
mean levels between groups but still remaining within the normal range were not supposed to be risky genes as well.

\section{The hierarchical clustering analysis}

To verify that the extracted DEGs can effectively differentiate between good and poor outcomes and whether groups of the same outcome can be further divided into subgroups, we applied the unsupervised hierarchical clustering analysis classification method.

We used all DEGs in the clustering analysis of 53 $\mathrm{BC}$ tumor tissue samples, the results of which are shown in Figure 2

Figure 2A, 2B shows that 14 of 15 recurrent patients (poor prognosis) clustered in the same cluster. Namely, 93\% of patients who experienced recurrence clustered together and significantly differed from patients who did not experience recurrence. This result showed that the obtained DEGs can predict prognosis in patients with BC. Notably, non-recurrent patients were also divided into two different subgroups. Group 1 contained 28 samples that exhibited the most significant difference from recurrent samples, which indicated the least risk of recurrence; Group 2 contained 9 samples that were most similar to recurrent samples, indicating a higher risk of recurrence. Thus, patients in Group 2 were identified to be at risk for recurrence.

\section{Identification of risk-associated pathways}

The hierarchical clustering analysis results show that the extracted DEGs can efficiently distinguish recurrent $\mathrm{BC}$ patients from $\mathrm{BC}$ patients. These genes may be involved in important biological functions that affect therapeutic outcomes. However, even patients with the same clinical phenotype exhibit disease heterogeneity. Moreover, the clustering analysis also identified different subgroups in the same group. These findings all indicate that functional level differences exist not only in patients with different prognoses but also in patients whose clinical signs are similar. To further analyze functional differences between patients whose risk of recurrence differs, we calculated the deviation score of 278 KEGG pathways using DEGs. Patients were classified according to the deviation scores based on up- or down-regulated DEGs (Supplementary Table S2). Finally, we selected four pathways with precision values greater than $65 \%$ as risk-associated pathways. These 4 pathways are listed in Table 1.

Because we also identified a small group of patients at risk for recurrence in the good prognosis group, we analyzed the relationship of these patients with those who had a different outcome. We show the performance of each pathway in Figure 3.
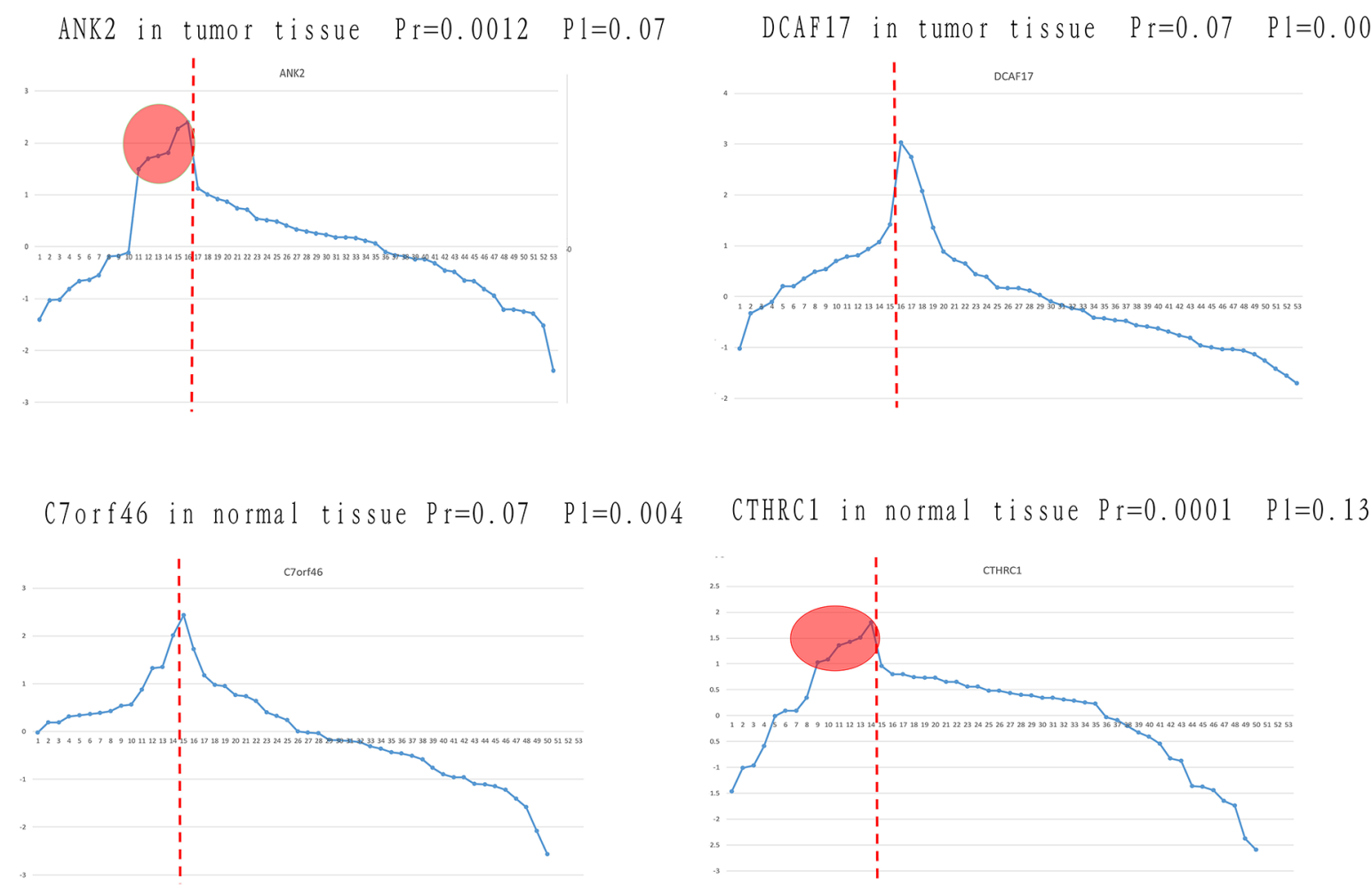

CTHRC1 in normal tissue Pr=0.0001 $\mathrm{Pl}=0.13$

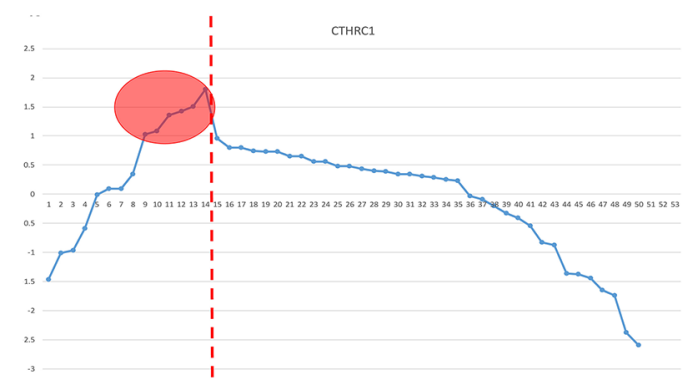

Figure 1: Identification of differentially expressed genes using our approach and traditional methods. The four genes that were not consistently identified using the traditional method and our method are listed. The horizontal axis represents samples, and the vertical axis represents gene expression. Samples on the left of the red line are from the poor prognosis group, and those on the right are from the good prognosis group. Pr and Pl represent the $p$ value obtained by randomization and the Limma algorithm, respectively. 
Table 1: Risk-associated pathways identified in tumor tissue

\begin{tabular}{lccc}
\hline \multicolumn{1}{c}{ pathway } & precise1 & precise2 & fuzzy \\
\hline hsa04020 calcium signaling pathway & 0.79 & 0.8 & 10 \\
hsa04540 Gap junction & 0.79 & 0.73 & 9 \\
hsa04010 MAPK signaling pathway & 0.74 & 0.73 & 9 \\
hsa04630 Jak-STAT signaling pathway & 0.74 & 0.8 & 11 \\
\hline
\end{tabular}

Precise 1 and 2 are the precisions of the good and the poor prognosis groups, respectively. Fuzzy represents intersected or isolated patients.

The activity of these four risk-associated pathways significantly varied in both patients with a good prognosis and with a poor prognosis. Therefore, the four pathways can effectively identify the recurrent risk of $\mathrm{BC}$ recurrence, with a prediction accuracy exceeding $65 \%$. Notably, nearly all patients at risk for recurrence were located in the middle of good and poor group patients. Even if at-risk patients do not experience recurrence, they resembled patients with a poor outcome at a functional level. When dysfunction of these risky pathway occurs, disease progression may happen on these risky patients.
Except for the isolated fuzzy patients, the ratio of risky patients overlapped with intersected fuzzy samples was from $33 \%$ to $56 \%$, which highlighted the use of functional pathway in distinguishing patients with diverse degree of recurrent risk.

To further identify the relationship of risk-associated pathways in normal status or tumor status, we searched for shared biological functions and activities. The function of inhibiting of apoptosis can be found in all of the four normal tissue related risky pathways. This finding indicated that apoptosis tends to be more inhibited in
A

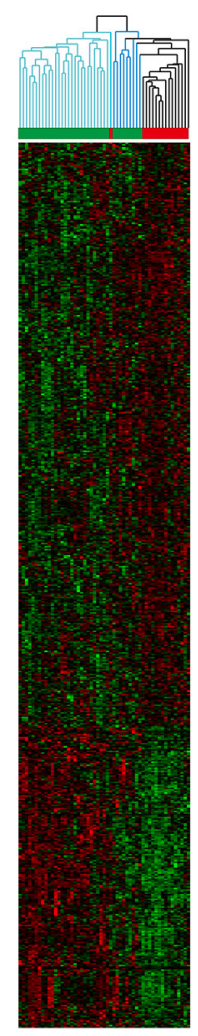

B

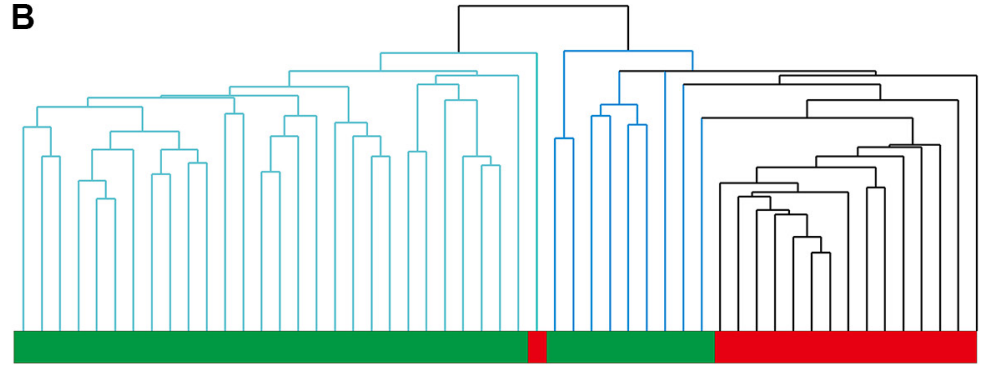

D

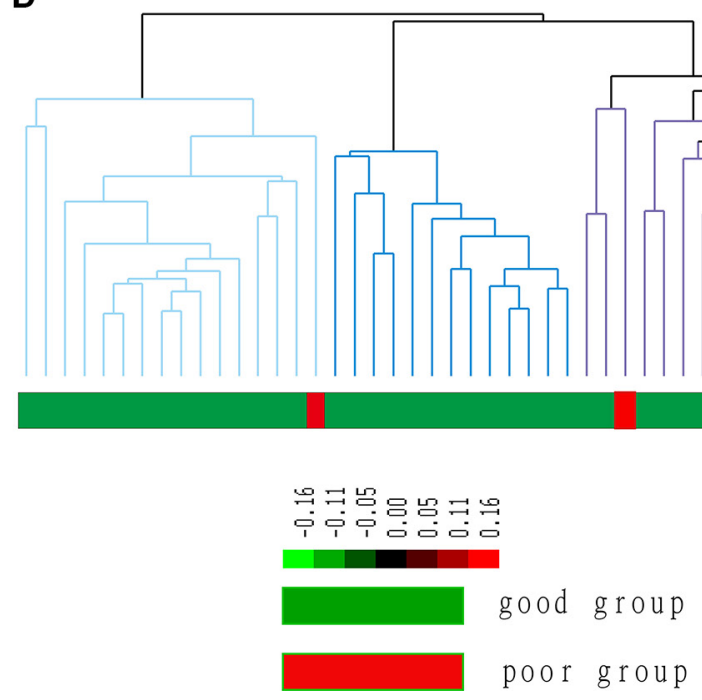

C

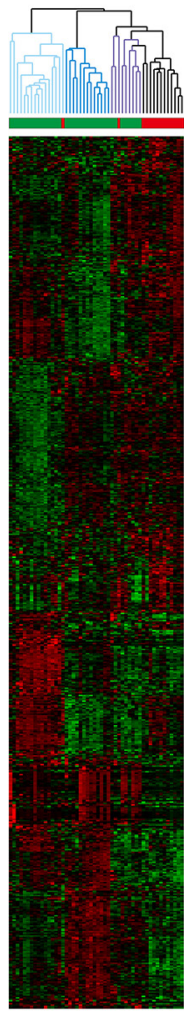

Figure 2: Cluster results for tumor and normal tissue samples. The good prognosis group indicates patients who did not experience recurrence, whereas patients in the poor prognosis group experienced recurrence. Groups 1 and 2 represent two subgroups of the good prognosis group in B; group 1 is marked in light blue, and group 2 is marked in dark blue. Groups 1, 2 and 3 represent three subgroups of the good prognosis group in $\mathrm{D}$, and group 3 is marked in purple. 
the nornal tissue of patients with poor outcomes than in other patients. The inhibition of apoptosis in normal cells leads to dysfunction due to aging and the accumulation of mutations, and the inhibition of tumor cell apoptosis results in aberrant cancer cell proliferation. Moreover, the four tumor status-related, risk-associated pathways also shared multiple biological functions, including apoptosis, proliferation, and the cell cycle. If the dysfunction in normal tissue represents the beginning of a loss of control, then blocking the cell cycle and activating proliferation may represent the associated process and consequence.

\section{Construction of a classifier model that integrates risk-associated pathways}

We integrated the risk-associated pathways derived from the tumor tissue analysis as features, and the random forest classifier reached an accuracy of $94 \%$, as shown in Figure 4. This result demonstrated the effectiveness of the risk-associated pathways in identifying recurrence risk and generated an improved model compared with the use of a single risk-associated pathway. To intuitionally observe differences among patients at the pathway level, we utilized a $3 \mathrm{D}$ coordinate system to visualize the patient distribution, as shown in Figure 5.

\section{Survival analysis based on risk-associated genes in an independent breast cancer dataset}

We extracted a total of 60 risk-associated DEGs from the four risk-associated pathways as risky genes and compared the survival of patients exhibiting expression level changes in at least one of these genes to that of patients not exhibiting any changes in these levels. Thirtyfive genes were removed because they were not related with survival. The results of the survival analysis based on the remaining 25 genes are shown in Figure 6.

\section{Normal tissue study}

\section{Analysis of differentially expressed genes}

To further investigate whether the physiological differences at the functional level in precancerous tissue can affect therapeutic outcome, we also analyzed the transcriptional profiles of normal tissue in patients with
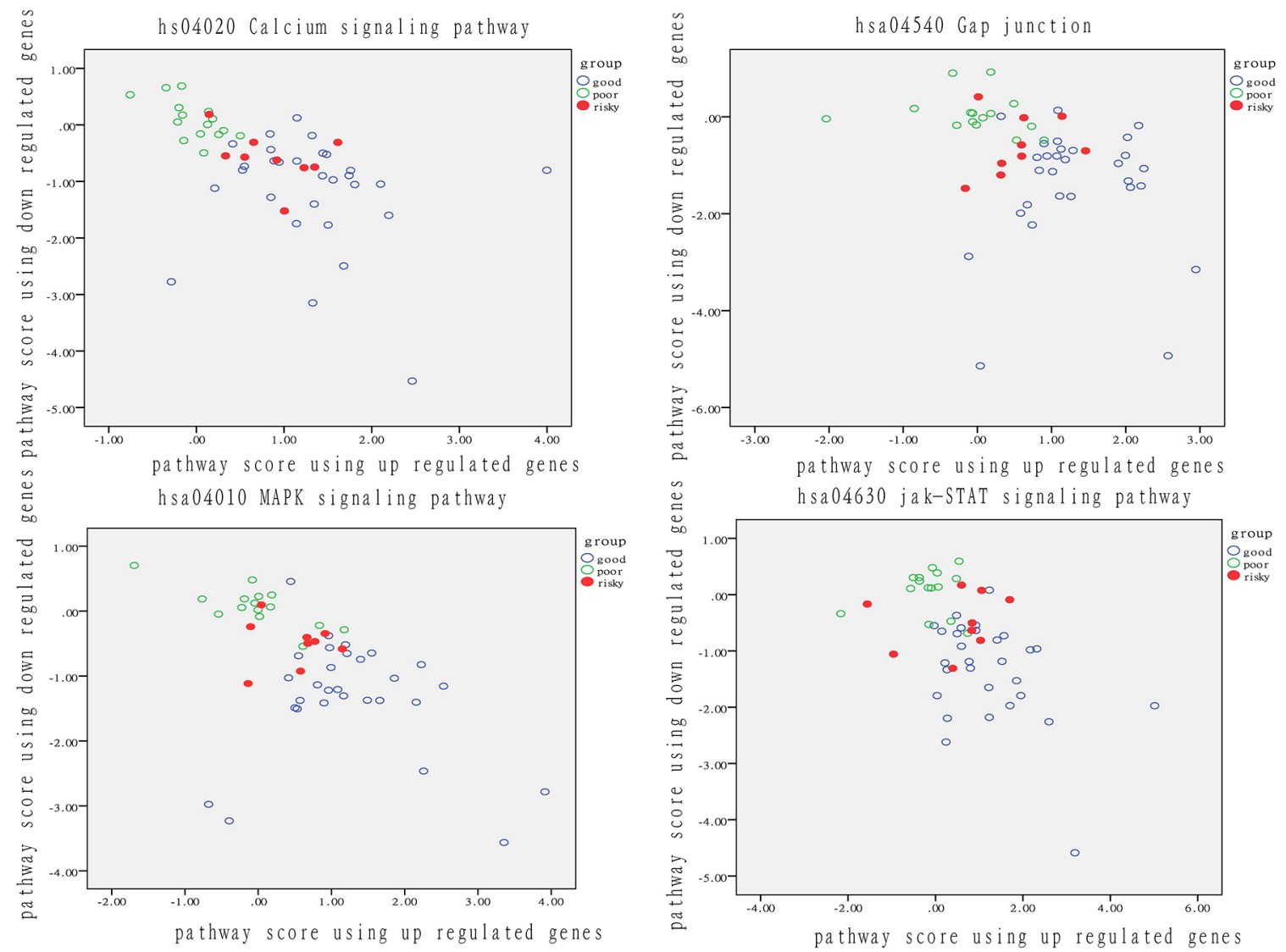

Figure 3: Analysis of patient distribution based on risk-associated pathways in tumor tissue. The horizontal axis represents the pathway deviation score based on up-regulated genes; the vertical axis represents the pathway deviation score based on down-regulated genes. The good prognosis group marked in blue represents patients who experienced a good outcome; the poor prognosis group marked in green represents patients who experienced a poor outcome. At-risk patients are marked in red. 


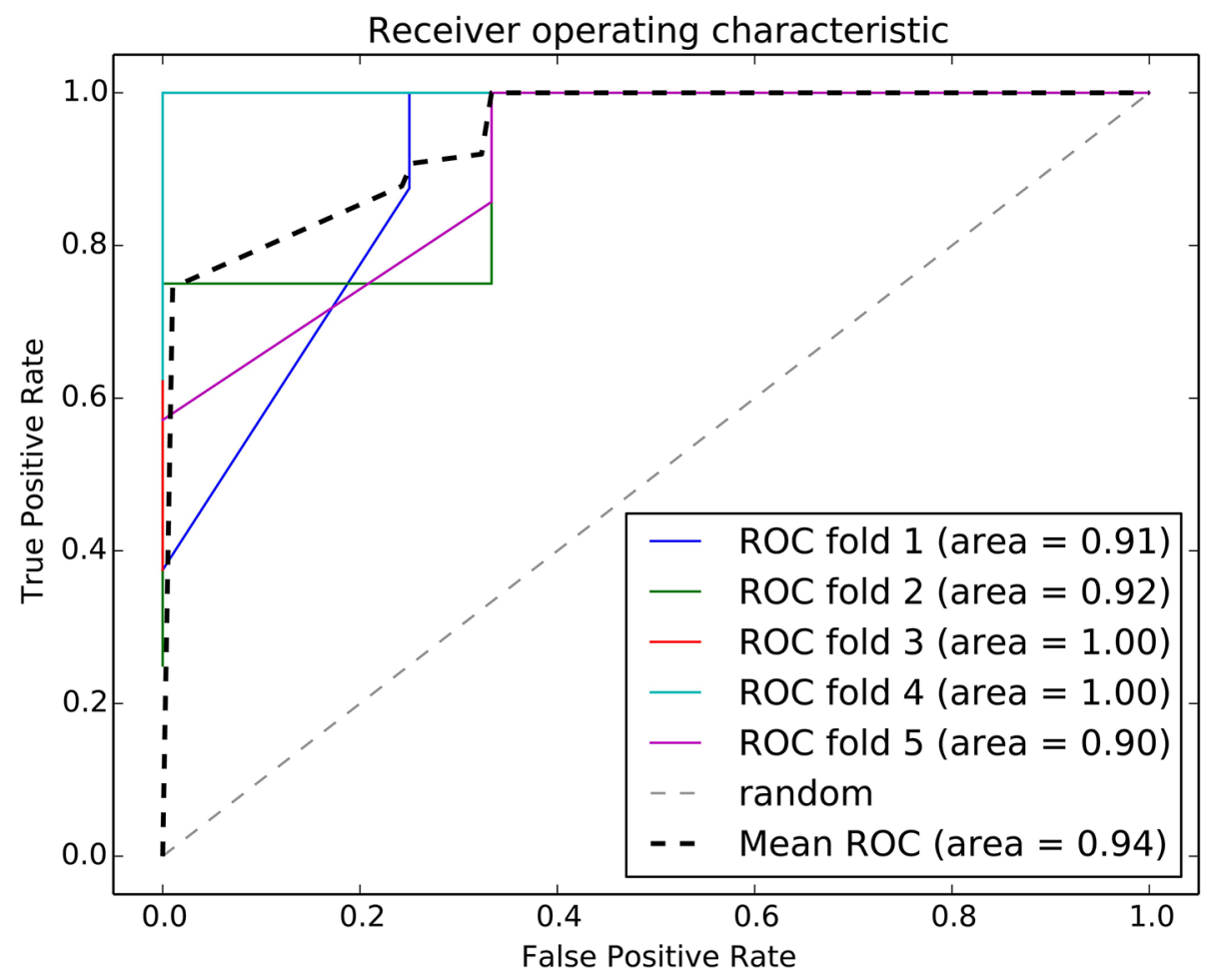

Figure 4: ROC curve of the random forest classifier using risk-associated pathways from tumor tissue. Five-fold cross validation was used to evaluate the model performance. Each iteration is marked in a different color, and the average ROC curve is marked in black.

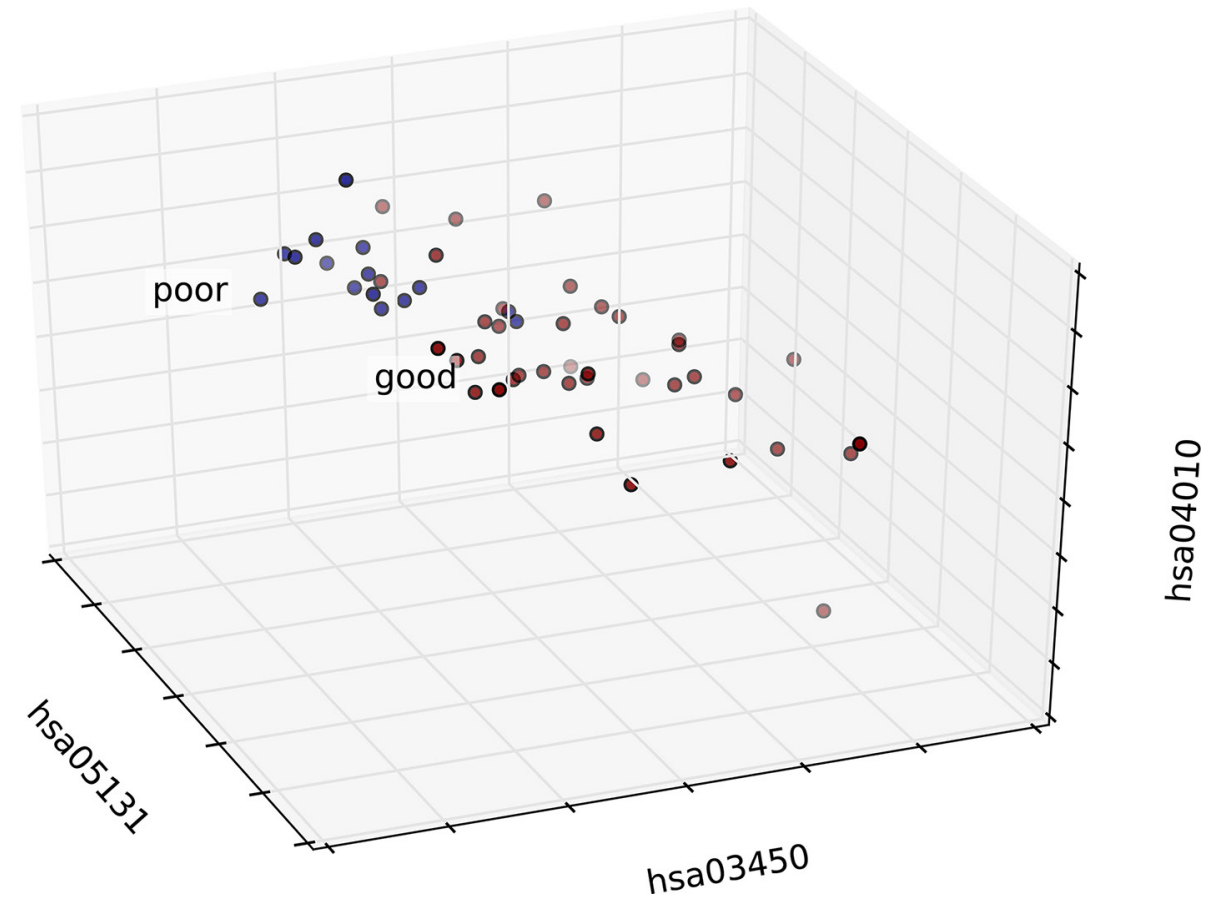

Figure 5: 3D graph of patient distribution based on risk-associated pathways in the tumor. Patients in the good and poor prognosis groups are marked in red and blue, respectively. The top three features were selected as axes, and each patient was mapped according to the values of these three features. 
good and poor prognoses. After FDR correction, we obtained 3134 genes, of which 1336 were up-regulated and 1798 were down-regulated genes (Supplementary Table S3).

\section{The hierarchical clustering analysis}

The hierarchical clustering result using all DEGs is shown in Figure 2C, 2D. Specifically, 12 of 16 patients who experienced recurrence were assigned to the same cluster, i.e., $75 \%$ of patients with recurrent $\mathrm{BC}$ differ from patients whose disease did not recur at the physiological level. In addition, the good prognosis group can be further divided into three subgroups (subgroup 1 is marked in light blue, subgroup 2 is marked in dark blue, and subgroup 3 is marked in purple). Subgroup1 contains 16 samples (15 from the good prognosis group and 1 from the poor prognosis group), subgroup 2 contains 18 samples (18 from the good prognosis group), and subgroup 3 contains 11 samples ( 8 from the good prognosis group and 3 from the poor prognosis group). Patients in subgroups 1 and 2 exhibited more apparent differences compared with the poor prognosis group, whereas patients in subgroup 3 were most similar to patients from the poor prognosis group. Thus, patients in subgroup 3 are at a risk of recurrence even though they mostly experienced good outcomes

\section{Identification of risk-associated pathways}

In nomor tissue, we also ranked the 278 pathways using DEGs according to their discrimination precision and we set $65 \%$ as threshold and got 4 pathways listed in Table 2 (Supplementary Table S4). The discrimination performance of each pathway is shown as in Figure 7.

These four pathways can effectively predict outcome. At-risk patients are marked in red and were much more similar to patients with a poor outcome than other patients in the good-outcome group. The overlap rate between at risk and fuzzy patients ranged from $29 \%$ to $71 \%$. These findings demonstrated that pathway activity also reflects significant differences in the normal tissues of patients. In other words, differences in normal tissue at the physiological level to some extent determine therapeutic outcome.

\section{Construction of a classifier model that integrates risk-associated pathways}

The risk-associated pathways derived from the normal tissue analysis were also used to construct a classifier model. An accuracy of $91 \%$ was achieved after a five-fold cross validation, as shown in Figure 8. The patient distribution based on the top three pathways is shown in Figure 9.

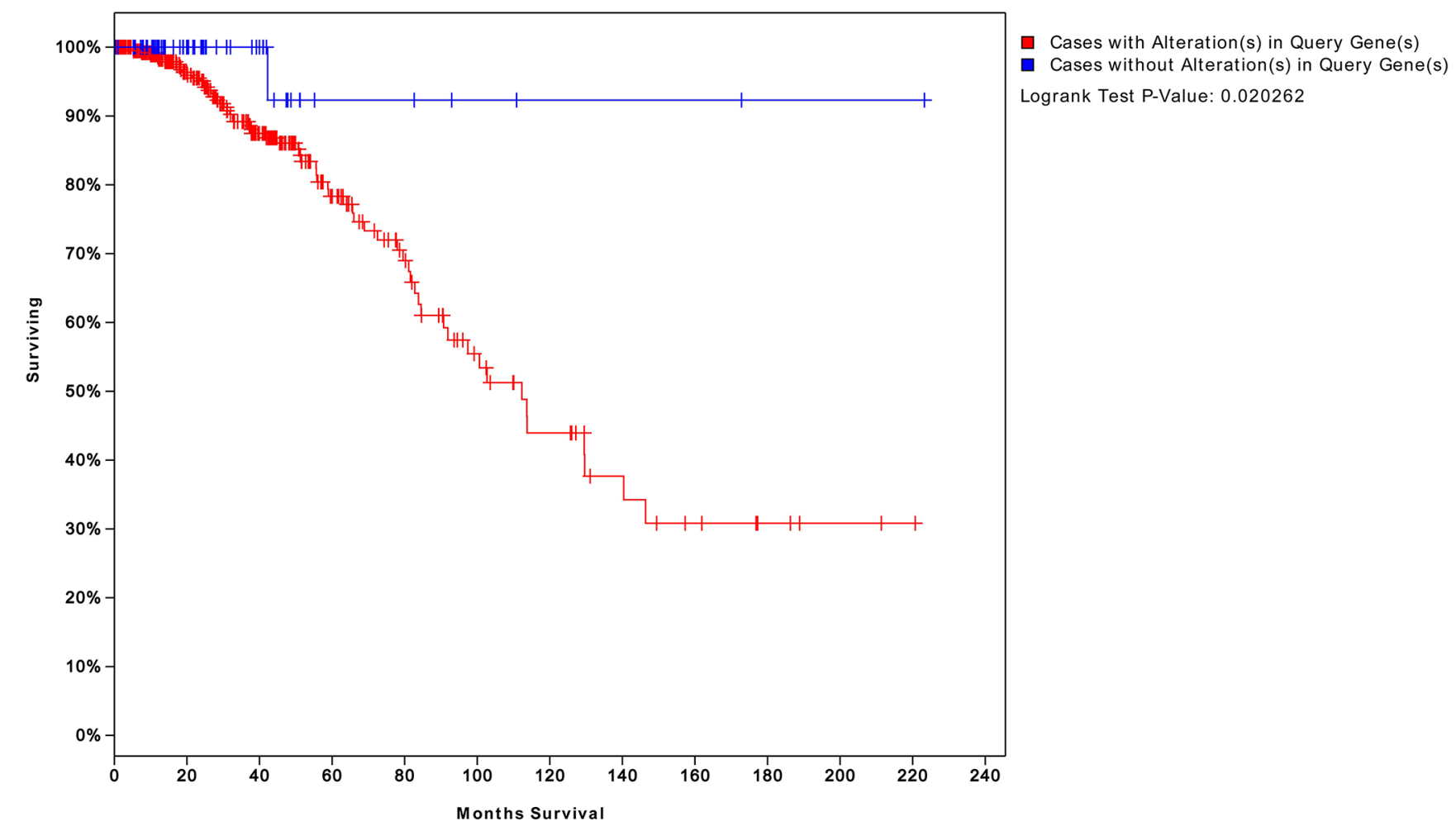

Figure 6: Survival analysis of patients with BC expressing genes risk-associated pathways in the tumor. The blue curve represents the survival time of patients with $\mathrm{BC}$ not exhibiting changes in the 25 risk-associated genes. The red curve represents survival time of patients exhibiting changes in at least one of these genes. A $P$ value of 0.02 indicates that a gene may serve as a biomarker to assess the risk of recurrence and survival time. 
Table 2: Risk-associated pathways identified in normal tissue

\begin{tabular}{lccc}
\hline \multicolumn{1}{c}{ pathway } & precise1 & precise2 & fuzzy \\
\hline hsa05152 tuberculosis & 0.74 & 0.75 & 8 \\
hsa05132 Salmonella infection & 0.67 & 0.69 & 13 \\
hsa05222 small cell lung cancer & 0.77 & 0.69 & 10 \\
hsa05164 influenza A & 0.79 & 0.62 & 11 \\
\hline
\end{tabular}

Precise 1 and 2 are the precision of the good and poor prognosis groups, respectively. Fuzzy represents intersected or isolated patients.

\section{Survival analysis based on risk-associated genes in an independent breast cancer dataset}

We identified 12 DEGs in these four risk-associated pathways and compared the survival time of patients with $\mathrm{BC}$ who did and did not exhibit changes in the expression of these genes.

We obtained four risk-associated pathways from the analysis of tumor tissue. These pathways can effectively predict patients with different prognosis,

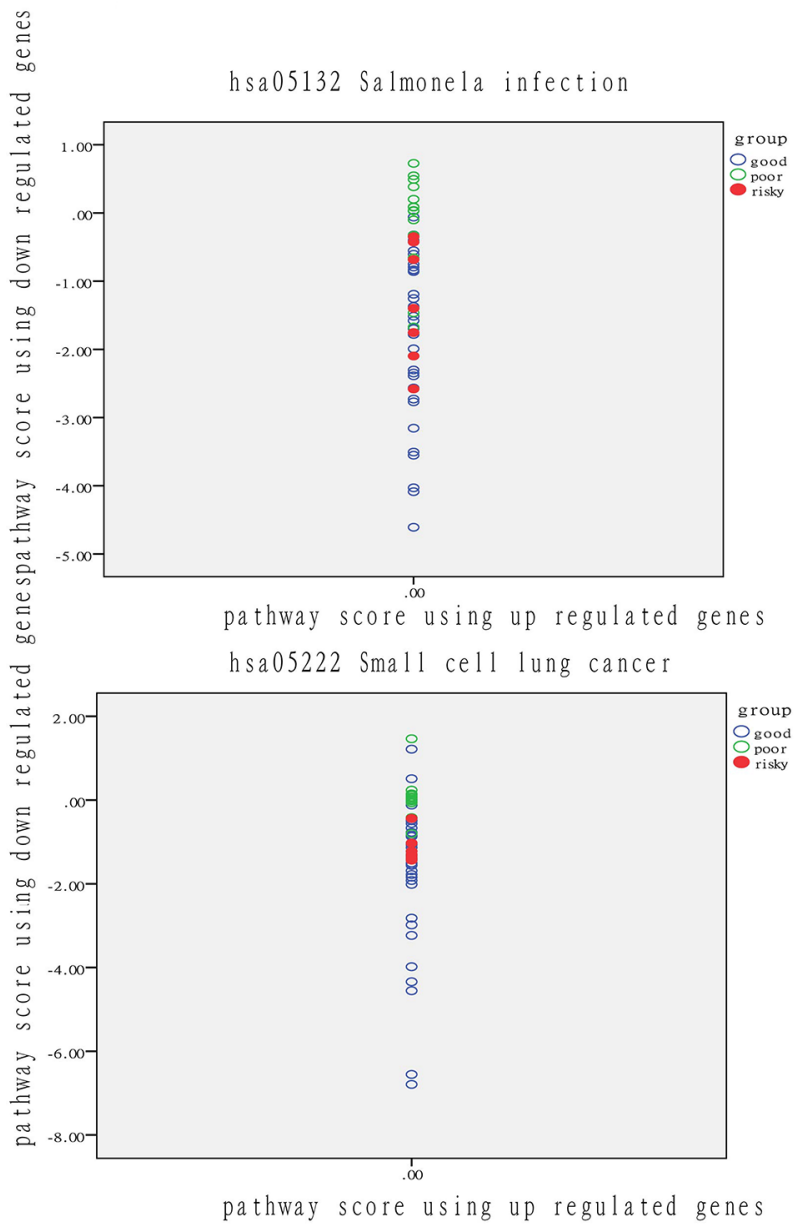

which demonstrated that the dysregulation of these four pathways may affect biological processes that influence prognosis. Moreover, we analyzed normal tissue from patients and identified four new risk-associated pathways that had not been identified in our analysis of tumor tissue. We integrated the genes from both normal and tumor tissue analyses to assess survival, and the final significance reached 0.003, as shown in Figure 10. The survival analysis suggested a significant relationship between riskassociated pathways and prognosis. Therefore, combining

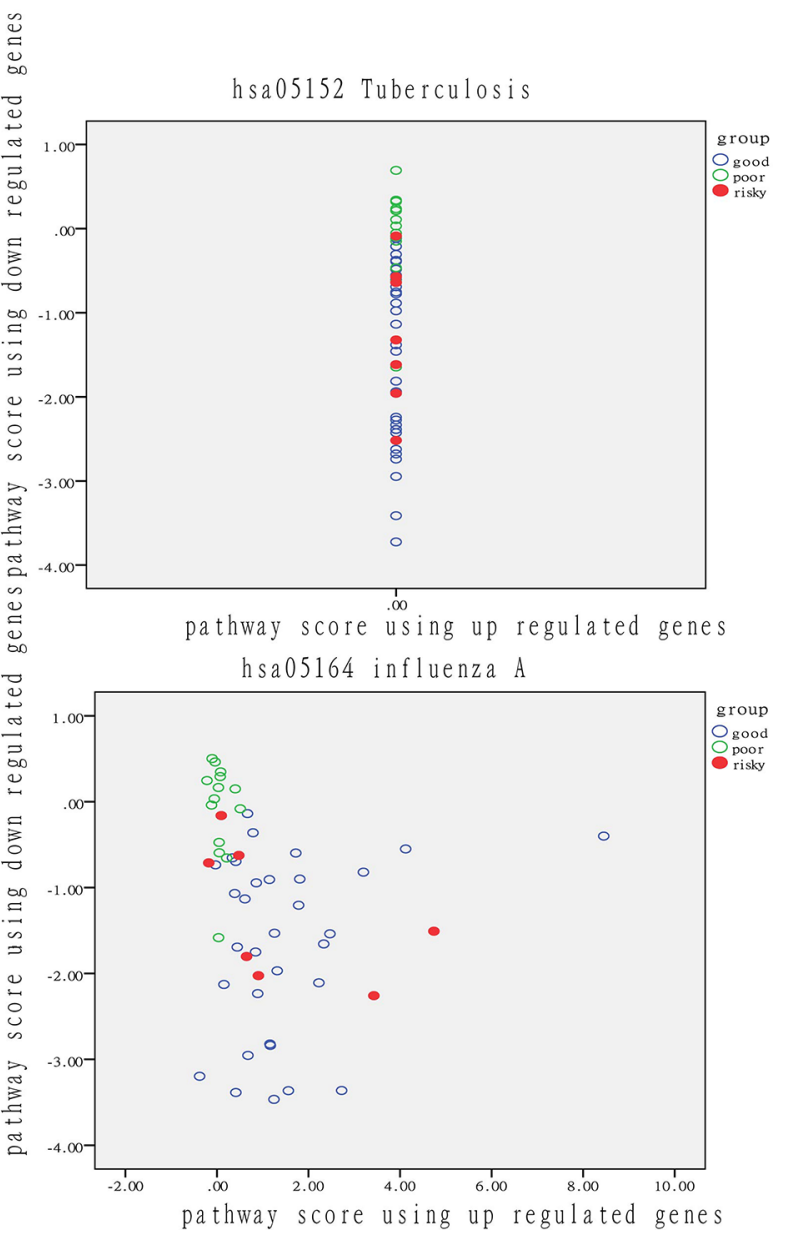

Figure 7: Analysis of patient distribution in normal tissue based on risk-associated pathways. The horizontal axis represents the pathway deviation score based on up-regulated genes; the vertical axis represents the pathway deviation score based on down-regulated genes. The good prognosis group marked in blue represents patients with a good outcome; the poor prognosis group marked in green represents patients with a poor outcome. At-risk patients are marked in red. 


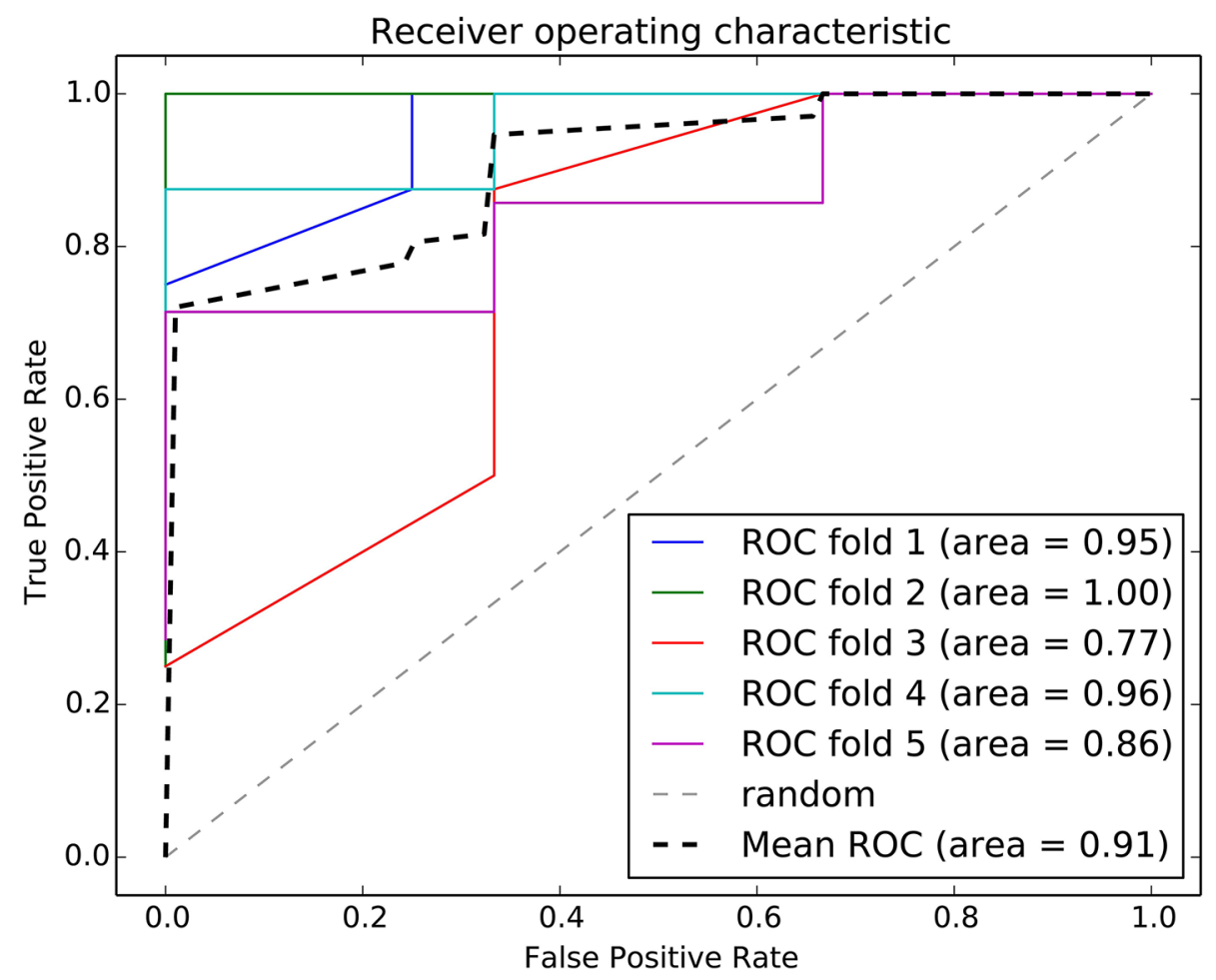

Figure 8: ROC curve of the random forest classifier using normal risk-associated pathways. Five-fold cross validation was used to evaluate the model performance. Each iteration is marked in a different color, and the average ROC curve is marked in black.

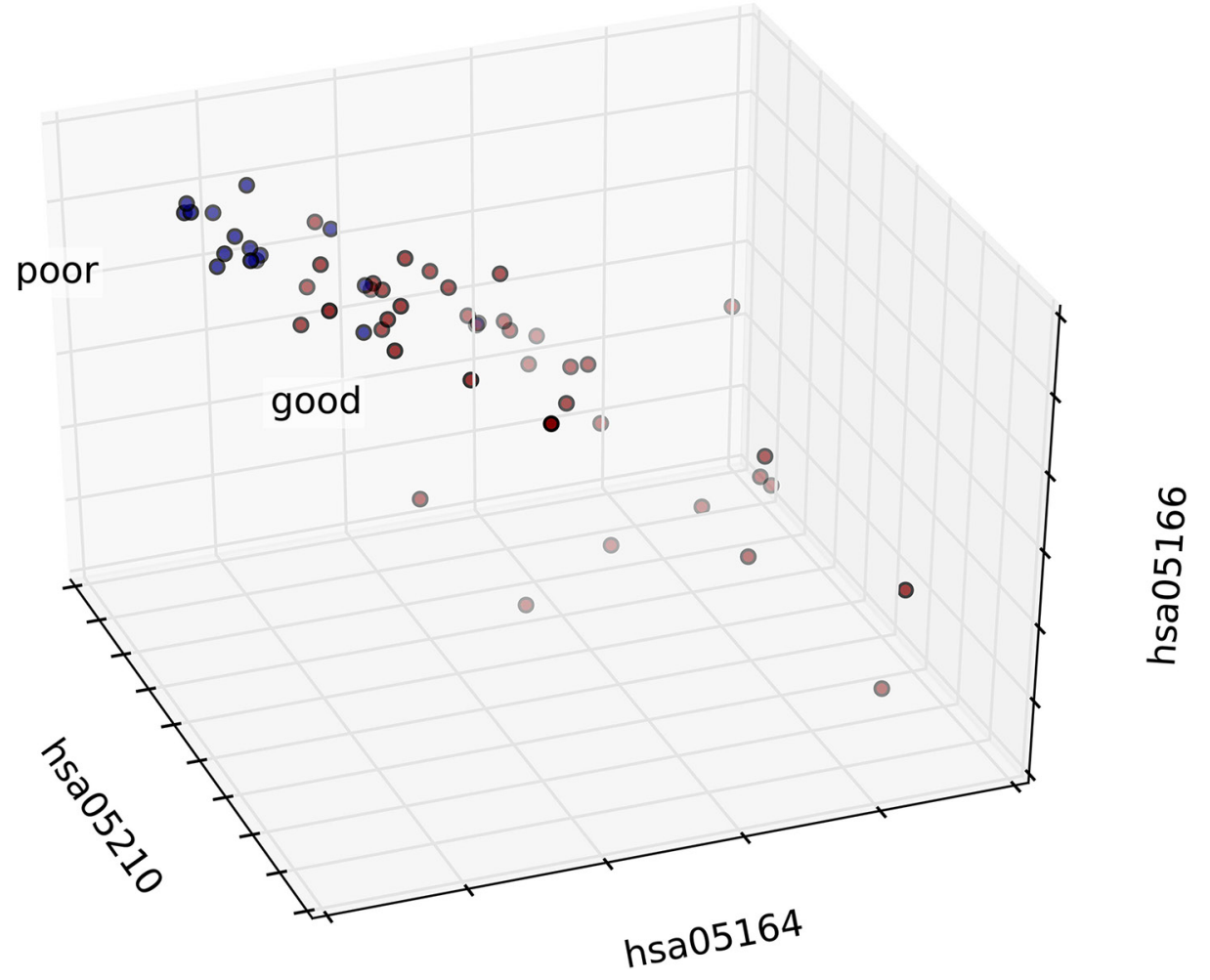

Figure 9: 3D graph of the patient distribution based on risk-associated pathways in normal tissue. Patients in the good and poor prognosis groups are marked in red and blue, respectively. The top three features were selected as axes, and each patient was mapped according to the values of these three features. 
analyses of normal and tumor tissue characteristics is of great significance for systematical and comprehensive cancer research.

\section{Classification of predictive models based on a cancer hallmark network framework}

Hallmarks of cancer depict the logical framework for conceptualizing the variety of neoplastic diseases. Over the past decades, the analysis on diversity of cancers based on the framework constructed by hallmarks had greatly promoted the understanding of occurrence, development and metastasis of different cancers. To discuss a recent idea of cancer hallmark network framework which is taking about constructing mechanism-based predictive models in cancer.We integrated risk-associated genes identified in normal and tumor tissue analysis and enriched to ten hallmarks [19] to finally obtaining specific hallmarks. We used an SVM classification model with the five-fold cross validation method to predict $\mathrm{BC}$ patients with recurrent risk and validate the performance of classification. The results are shown in Figure 11. The classification performance was highest for the integrated hallmark risk gene model, with an AUC of $92 \%$.

At $p<0.05$, risk-associated genes expressed in normal tissue were significantly enriched in five hallmarks, that is Resisting Cell Death (RCA),Insensitivity to Antigrowth
(IA), Limitless Replicative Potential (LRP),Self Sufficiency in Growth Signals (SSGS) and Sustained Angiogenesis (SA), whereas the significantly enriched hallmarks by riskassociated genes expressed in tumor tissue were IA and SSGS (Supplementary Table S5). Based on the enrichment results,we found that the normal tissue obtained the most fundamental trait of cancer cells,RCA,IA and SSGS, which represented the underlying molecular mechanisms to sustain chronic proliferation, resist to cell death,and resist inhibitory signals that might prevent their growth [20]. while sustaining Angiogenesis represents underlying molecular mechanisms for angiogenesis, which represents stimulating the growth of blood vessels to supply nutrients to tumors and evacuate metabolicwastes [20]. Namely, for the corresponding normal tissue of the patients with poor prognosis, it has lost its homeostasis due to loss the normal regulation to cell proliferation, differentiation and death under some tumorigenic factors, which made the normal tissue cells obtain the ability to survive without external environment stimulation, and angiogenesis ability for infinite differentiation cells to provide the necessary nutrients. That showes these hallmarks are related to preliminary stage of cancers $[21,22]$. Therefore, "normal cells" exhibiting the above hallmarks will eventually develop into new cancer cells under certain conditions to result in recurrence even if the tumor was removed and the patient received adjuvant systemic therapy.

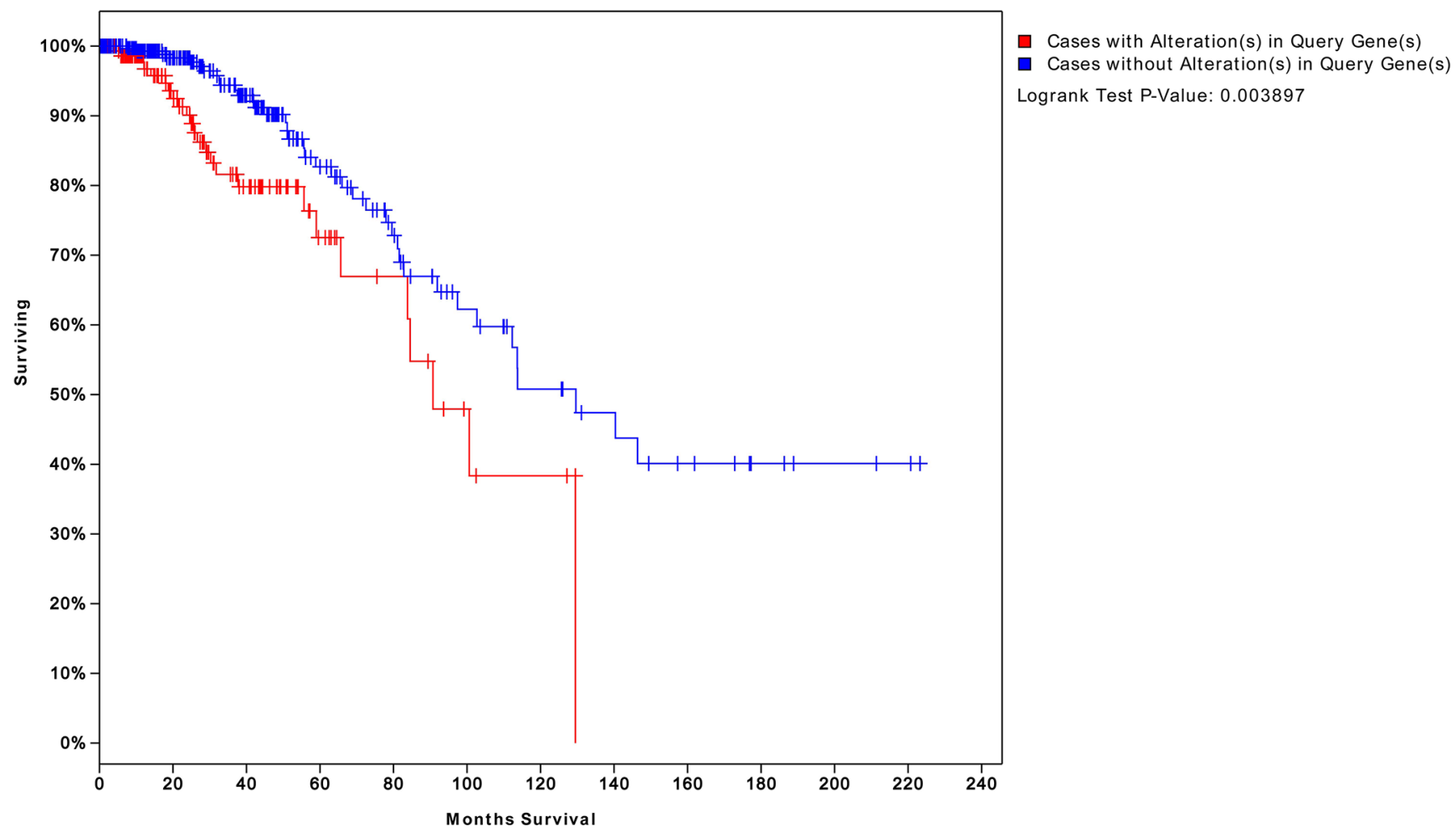

Figure 10: Survival analysis integrating both normal and tumor pathway genes. We integrated the risk-associated genes from both normal and tumor tissues. Survival significantly differed between patients who did and did not exhibit changes in the expression levels of these genes $(P=0.003)$. This result suggests that integrating normal and tumor tissue analyses can better predict patient prognosis than an analysis of either status alone. 


\section{DISCUSSION}

Breast cancer is a highly heterogeneous cancer and treated with different therapy strategies based on its clinical immunohistochemical classification. However, as the number of patients experiencing resistance, recurrence and metastasis increases, increasing efforts have focused on the heterogeneity of breast cancer patients at the molecular level. In recent years, many studies have identified biomarkers related to the prognosis of breast cancer based on feature extraction, classifier, regression, and other statistical methods. However, most studies have relied on identifying common molecular features involved in the prognosis of patients at the pathological level and did not consider differences among breast cancer patients at the physiological level. We hypothesized that in addition to differences at pathological level in carcinoma tissue, physiological

A

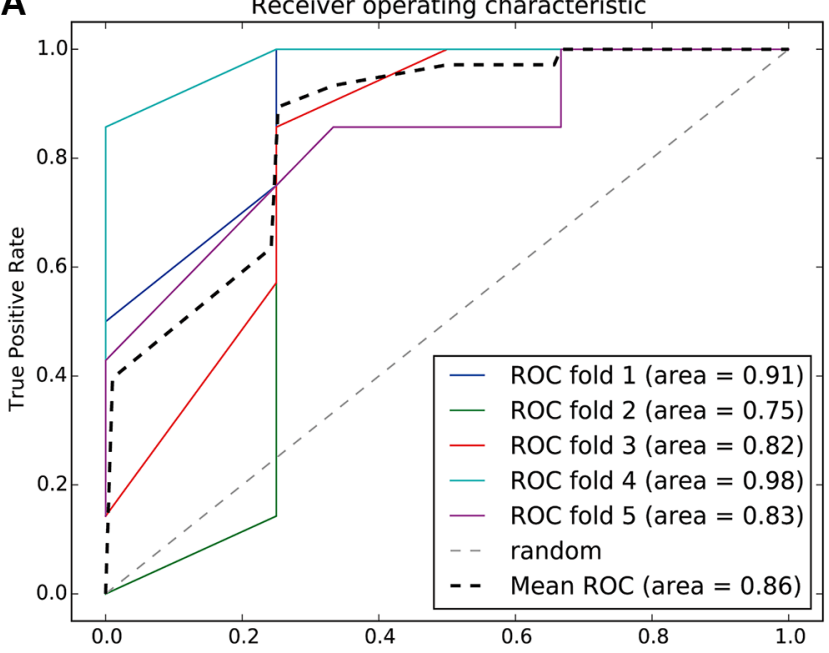

C

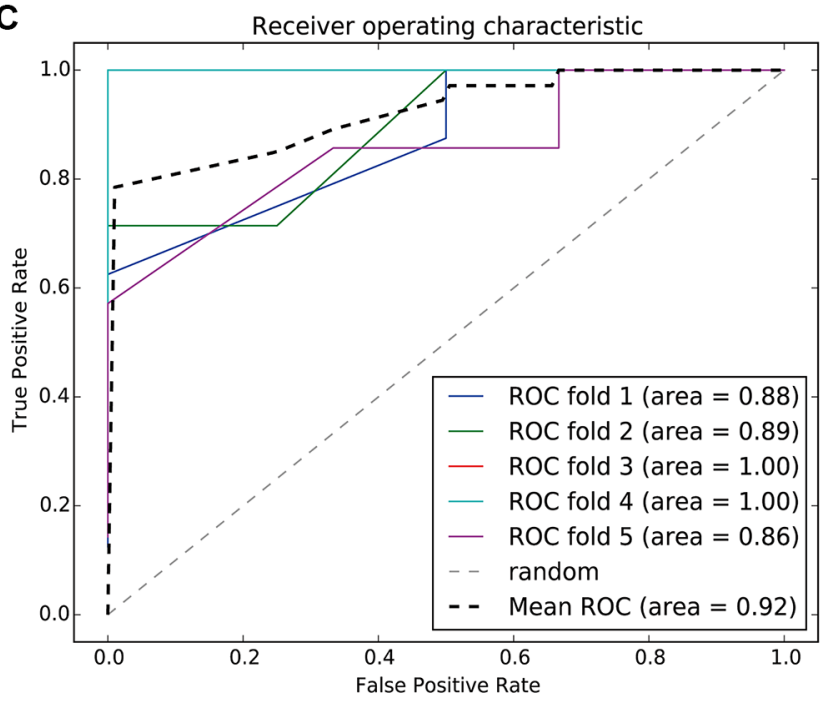

level differences in the normal tissue of patients may also contribute to the risk of recurrence or metastasis.

Considering the heterogeneity of breast cancer, we calculated the normal ranges of gene expression based on the levels measured in non-recurrent patients instead of comparing the mean expression levels between two groups. Using randomization, we identified specific genes that were only altered in subgroups and likely reflect individual recurrence risk by participating in the mechanisms of recurrence. Our method identified specific genes omitted by traditional methods. These genes may only be differentially expressed in small groups; thus, changes in these genes are usually less significant when compared to the mean values of background data. In addition, some genes whose mean expression levels differed between two groups according to traditional methods were also regarded as nonspecific genes because

B

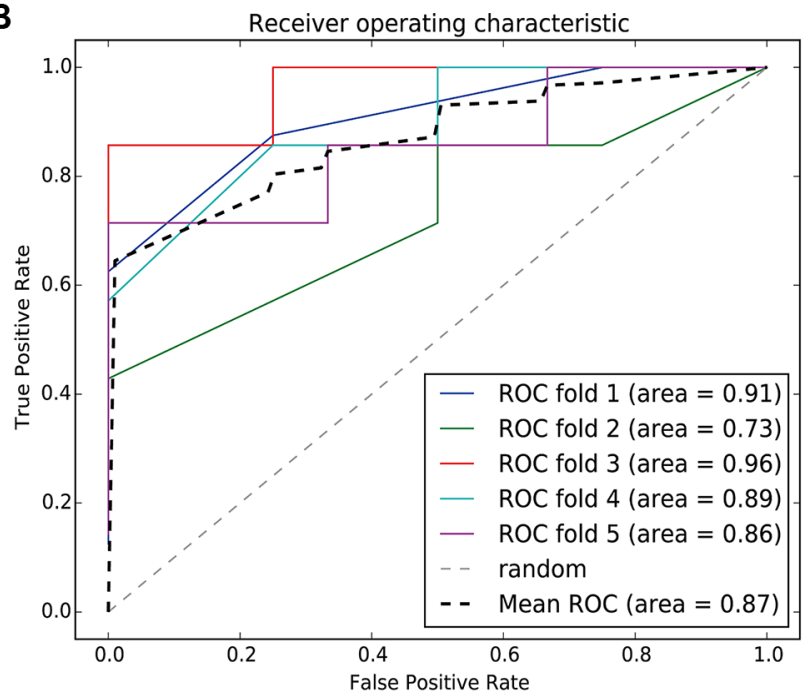

Figure 11: ROC curve of the SVM classification model using risk-associated genes in normal tissue ,tumortissue and both tissue. Each iteration is marked in a different color, and the average ROC curve is marked in black. (11A-11C) represents, respectively, the ROC curve of the SVM classification model using risk - associated genes from normal tissue, tomor tissue and integerated both tissues. 
they lacked significance after randomization. Although the mean expression levels of these genes differed between two groups, these levels fluctuated within normal ranges. A hierarchical cluster analysis showed that the genes we identified can effectively predict prognosis. Moreover, even patients with the same prognosis phenotype can also be subdivided into different subgroups, which demonstrated that our method can efficiently identify different risk-associated subgroups and reflect personalized characteristics.

We used both the normal and tumor tissues of patients with diverse outcomes for analysis and identified eight risk-associated pathways, all of which are related to immune regulation and drug response. Our findings demonstrate a remarkable relationship between risk-associated pathways and the prognosis of patients with $\mathrm{BC}$ in terms of both recurrence risk and survival time. The risk-associated pathways extracted from tumor and normal tissues mediate various biological functions. Mahmood $\mathrm{M}$ found that combining a calcium-channel blocker with an alternative delivery method for the anti-cancer drug reduced cellular resistance to chemotherapeutics [23]. Gielen PR showed that increasing gap junction protein expression in human glioma cells enhanced resistance to TMZ treatment [24]. Moreover, a number of cancer-related biological processes, such as the apoptosis of leukemic cells [25] and oxidative stress resistance [26], are mediated by the MAPK signaling pathway, and the hyperactivation of this pathway can cause drug resistance [27]. BecerraDíaz M highlighted the importance of the JAK-STAT signaling pathway in regulating drug susceptibility and resistance by studying mice deficient in STAT molecules [28]. The other four risk-associated pathways identified normal tissue are all involved in infection and inflammation, such as the intrinsic immune response [29-32]. Therefore, we speculate that both the intrinsic immune system in normal tissue and the drug response in tumor tissue can influence prognosis of patients with BC. Combining analyses of tumor and normal tissue can help to comprehensively identify cancer prognosis-related functions. Invaluable information may be missed when focusing on only on tumor or normal tissue. Specifically, survival analyses were much more significant when riskassociated genes from both normal and tumor tissue were integrated compared with either single gene set alone.

Intriguingly, the four pathways most significantly differed in normal tissue between the good and poor prognosis groups, and this difference was less significant in cancer tissues. Similarly, the four most significant pathways in cancer tissues were less significant normal tissue. This finding suggests that significant changes occur at both the physiological and pathological levels during malignant transformation. Certain genes or functions significantly differed in normal tissue based on patient outcomes, and these genes and functions were associated with the immune response, cell replication, drug sensitivity and other related functions. These genes and functions exhibit dysfunction during malignant transformation. Therefore, inherent differences at the physiological level are masked, and differences at pathological level are dominant. Thus, we speculate that significant differences in the genes and functions in normal tissue determine the inherent risk of cancer development, and differences in genes and functions in tumor tissue may explain the relationship between acquired dysfunction and recurrence. Combining the inherent and acquired risk factors for breast cancer recurrence may serve as a more comprehensive method for evaluating the prognosis of patients.

All four risk-associated pathways in normal tissue were involved in the inhibition apoptosis, which could be considered the first step of malignant transformation. Aging and the continuous accumulation of mutations result in the dysfunction of normal cells and even their transformation into tumor cells, which is followed blocking the cell cycle and activating proliferation in tumor tissue. Dysfunction in this second stage hinders the killing of cancer cells because of their increased proliferation, whereas normal cells are rendered fragile. This finding demonstrated the importance of identifying cancerous characteristics in normal tissue early. Specifically, the degree of apoptosis inhibition in normal tissue directly correlates with the risk of recurrence or drug resistance because normal cells are more fragile and easier to transform into tumor cells.

In addition to the risk of recurrence itself, we also evaluated prognosis based on the survival time to identify genetic biomarkers that can effectively assess recurrence and survival time. We used genes extracted from riskassociated pathways analyze survival in patients with breast cancer based on mRNA data from TCGA. A KM survival analysis showed that the genes extracted from risk-associated pathways in normal tissue and tumor tissue can effectively predict survival time. These findings all demonstrated that patients with differentially expressed risk-associated genes have a distinctly shorter survival times than patients without any alterations in these genes. Assessing the risk of recurrence can objectively identify the value of chemo- or radiotherapy to patients to ultimately provide a personalized rational therapeutic schedule, which consequently reduces the side effects of radiation and chemotherapy and improves the cure rate.

Classification based on risk-associated pathways also identified fuzzy samples that exhibited intersection or isolation. These patients failed to be classified into any group based on risk-associated pathways. The emergence of these fuzzy samples (isolation) was partly due to differences in personalized gene expression and bifurcation point data, which are usually difficult to distinguish. The other type of fuzzy sample (intersection) exhibited significant overlap with at-risk patients in the clustering analysis. Moreover, age, gender, and health background may also impact feature extraction and 
classification. Thus, our future work will integrate more patient data and prognosis-related features to provide a more comprehensive prediction method.

\section{MATERIALS AND METHODS}

\section{Breast cancer patient data}

We selected 53 breast cancer (BC) samples that had transcriptional information for both normal tissue and tumor tissue from the TCGA database. According to clinical data, the samples were divided into the good prognosis group without recurrence (38) and the poor prognosis group, in which disease recurred (15). An independent dataset containing mRNA data from $526 \mathrm{BC}$ patients was used to analyze survival time [14]. The cBioPortal database was used to generate a K-M Survival curve using risk-associated genes [15].

\section{Differential expressed gene analysis}

We standardized the expression profiles. To eliminate inherent variations in gene expression, we used the Z-score correction method [16]. We defined the good prognosis group as the control group, and the poor prognosis group as the case group.

A normal interval was calculated based on the distribution of expression values in the good prognosis group (means \pm 1.96 standard deviations). Gene expression outside the normal interval may result in a poor outcome. For a gene G, the number of patients in the good prognosis group is $\mathrm{n} 1$, the number of patients in the poor prognosis group is $n 2$, and an initial score for $\mathrm{G}$ is calculated using the following formula:

$$
\text { score }=\sum_{i=1}^{n 2}\left(X_{2 i}-X^{\prime}\right)
$$

where

$$
X^{\prime}=\left\{\begin{array}{l}
X_{\max } \text { if } X_{2 i}>X_{\max } \\
X_{2 i} \text { if } X_{\min }<X_{2 i}<X_{\max } \\
X_{\min } \text { if } X_{2 i}<X_{\min }
\end{array}\right.
$$

$\mathrm{X}_{2 \mathrm{i}}$ is the expression value of the ith patient in the poor prognosis group, and $\mathrm{X}_{\max }$ and $\mathrm{X}_{\min }$ are two extreme values for the normal interval. Based on the sum of the expression of gene $\mathrm{G}$ outside the normal interval in poor prognosis group, the initial score of gene $\mathrm{G}$ can be calculated.

After initial score of $\mathrm{G}$ was obtained, all samples were randomly permutated, and samples with the number $\mathrm{n} 1$ were randomly allocated to the good prognosis group, whereas the remaining $\mathrm{n} 2$ samples were allocated to the poor prognosis group. A new random score was obtained using this formula, and the above process was repeated 10000 times. After permutation, the gene score background distribution can be translated into $P$ values; genes with a $P<0.05$ were considered DEGs.

\section{The hierarchical clustering analysis}

To prove that the DEGs we identified not only predict prognosis but also characterize the individual characteristics of small subsets of patients, we used the DEGs in the poor prognosis group to conduct a hierarchical cluster analysis of all samples. Furthermore, the cluster performance was assessed according to the sample label (good or poor outcome) of samples in each cluster. Expression profile data were filtered and standardized. The filtering process selected genes that are expressed in at least $80 \%$ of the sample with standard deviations greater than 1 . The normalization of both genes and the sample relied on the median standardization center method, and the similarity matrix was based on the correlation-centered method. The cluster 3.0 software was used for standardization and hierarchical clustering [17].

\section{Risk-associated pathway identification}

We downloaded 278 pathways from the KEGG database and assigned a score for each pathway based on the DEGs. Abnormal pathway function is not only reflected in the gene expression but also in the loss of balance in a pathway [18]. To identify pathway dysfunction due to imbalance, we calculated the pathway deviation score using up- and down-regulated genes. For pathway P, the number of up-regulated genes was N1, the number of down-regulated genes was $\mathrm{N} 2$, and the score vector $T=(U, D)$ can be obtained using formula 2 for sample $\mathrm{S}$.

$$
\begin{gathered}
U=\frac{\sum_{i=1}^{N 1}\left(x_{i}-\mu\right)^{3}}{N 1} \\
D=\frac{\sum_{j-1}^{N 2}\left(x_{j}-\mu\right)^{3}}{N 2}
\end{gathered}
$$

For a pathway $\mathrm{P}, U$ is the deviation score obtained using up-regulated genes, and $D$ is the deviation score obtained using down-regulated genes. $\mathrm{Xi}$ is the gene expression value in sample $S, \mu$ is the mean value in the good prognosis group, and $\mathrm{T}$ is the score vector for sample $\mathrm{S}$.

Finally, we obtained a score vector matrix of 53278 entries. The identification of risk-associated pathways, which can effectively distinguish between two groups of samples, is beneficial for early diagnosis and predicting prognosis. We first found the geometric center of samples in the good and poor prognosis groups and then classified each sample according to its distance from each geometric center. In this study, the geometric center, $\mathrm{O}$, was calculated using the score vector of samples in same group, and the radius, $\mathrm{R}$, was then assigned. The circle centered on $\mathrm{O}$ with radius $\mathrm{R}$ had to include at least $80 \%$ of 
samples in the same group. Specifically, $80 \%$ was set as the threshold to consider the heterogeneity among samples and prevent outliers. We then calculated the classification accuracy according to the percentage of correctly classified/predicted samples, as shown in Figure 12.

The classification accuracy was evaluated based on the proportion of observed samples in all predicted samples. Samples circled in red were classified as fuzzy (fuzzy sample) and included two types of samples, intersection and isolation. To ensure the accuracy of the diagnosis, these fuzzy samples were not assigned to any group. The top four pathways with an average accuracy exceeding $65 \%$ were considered risk-associated pathways.

\section{Building a classifier model that integrates risk-associated pathways}

We proposed an original method to evaluate the ability of each risk-associated pathway to predict outcome. We then integrated these risk-associated pathways to build an efficient classifier model using the random forest algorithm. Five-fold cross validation was used to evaluate the model efficiency, and the top three pathway features were used as axes in the 3D visualization analysis.

\section{Recognition of diagnosis biomarkers based on risk-associated pathways}

We extracted DEGs from risk-associated pathways, which can act as biomarkers to effectively identify patients at a high risk of recurrence. However, only predicting the risk of recurrence is insufficient to evaluate therapeutic outcomes because the survival time is another important evaluation criterion. Thus, we compared the survival time of 526 patients with BC from the TCGA dataset who did and did not exhibit changes in risk-associated pathway genes. To optimize and screen for significant biomarkers, genes that did not influence the survival analysis $p$ value were removed.

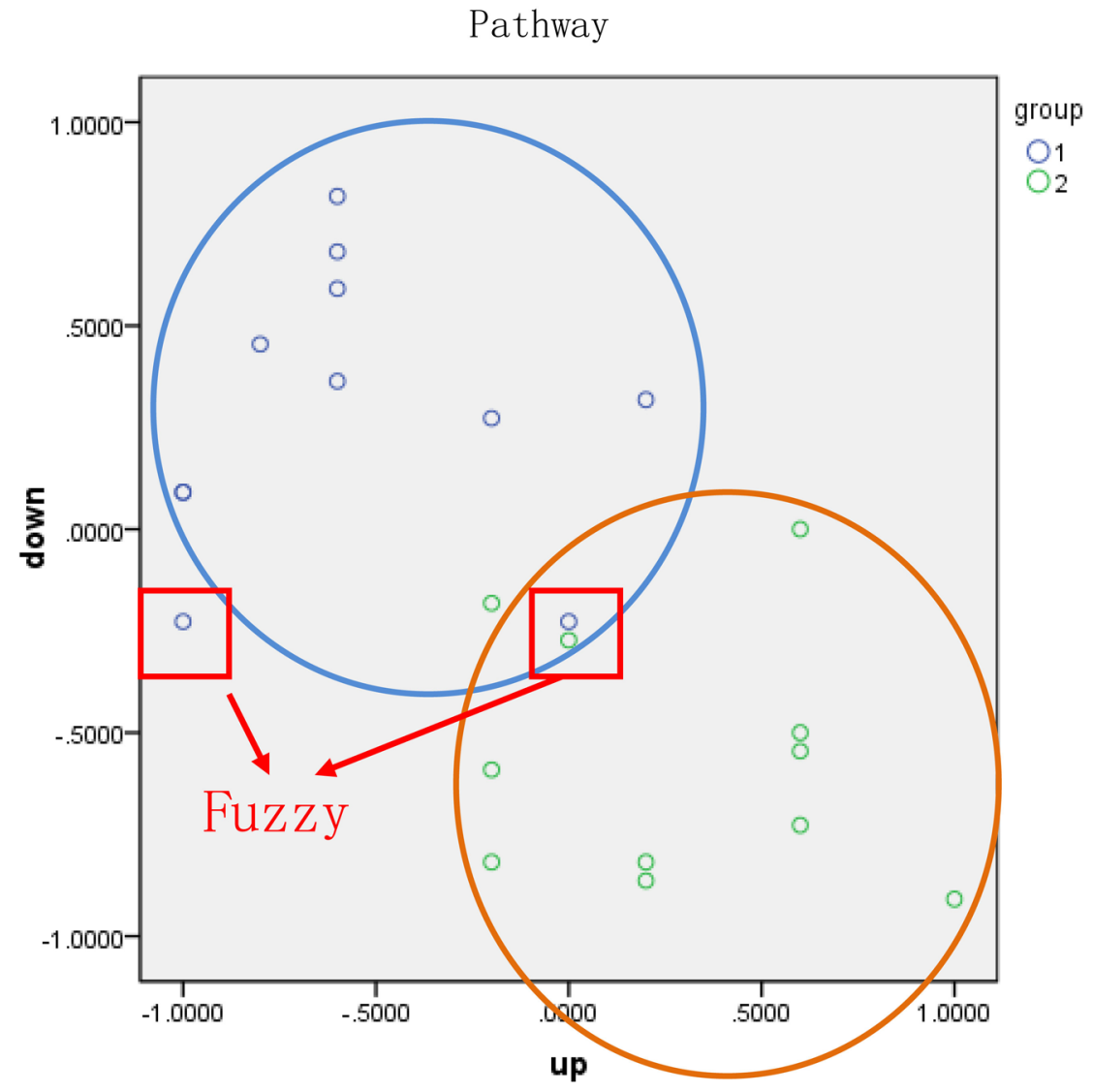

Figure 12: Classification of samples based on functional pathway. Blue and green points represent two types of samples. The horizontal and vertical axes represent the pathway score calculated using up- and down-regulated genes, respectively. The blue and orange circles were constructed based on the geometric center and the radius, respectively, of each group. 


\section{ACKNOWLEDGMENTS AND FUNDING}

The authors thank the National Natural Science Foundation of China [Grant No. 61372188], the Provincial Education Department Project of Heilongjiang, China [Grant No. 12541331], and the Graduate Innovation Foundation of Harbin Medical University, China [Grant No. YJSCX201542HYD and YJSCX2014-19HYD] for the supporting funds.

\section{CONFLICTS OF INTEREST}

The authors declare no other competing interests.

\section{REFERENCES}

1. Abe O, Abe R, Enomoto K, Kikuchi K, Koyama H, Masuda H, Nomura Y, Sakai K, Sugimachi K, Tominaga T, Uchino J, Yoshida M, Wilcken N, et al. Effects of chemotherapy and hormonal therapy for early breast cancer on recurrence and 15-year survival: an overview of the randomised trials. Lancet. 2005; 365:1687-1717.

2. Weng Ng, Delaney GP, Jacob S, Barton MB. Estimation of an optimal chemotherapy utilisation rate for breast cancer: setting an evidence-based benchmark for the bestquality cancer care. European journal of cancer. 2010; 46:703-712.

3. Goldhirsch A, Wood WC, Gelber RD, Coates AS, Thurlimann B, Senn HJ. Progress and promise: highlights of the international expert consensus on the primary therapy of early breast cancer 2007. Annals of oncology. 2007; 18:1133-1144.

4. Eifel P, Axelson JA, Costa J, Crowley J, Curran WJ Jr, Deshler A, Fulton S, Hendricks CB, Kemeny M, Kornblith AB, Louis TA, Markman M, Mayer R et al. National Institutes of Health Consensus Development Conference Statement: adjuvant therapy for breast cancer, November 1-3, 2000. Journal of the National Cancer Institute. 2001; 93:979-989.

5. Blamey RW, Ellis IO, Pinder SE, Lee AH, Macmillan RD, Morgan DA, Robertson JF, Mitchell MJ, Ball GR, Haybittle JL, Elston CW. Survival of invasive breast cancer according to the Nottingham Prognostic Index in cases diagnosed in 1990-1999. European journal of cancer. 2007; 43:1548-1555.

6. Ravdin PM, Siminoff LA, Davis GJ, Mercer MB, Hewlett J, Gerson N, Parker HL. Computer program to assist in making decisions about adjuvant therapy for women with early breast cancer. Journal of clinical oncology. 2001; 19:980-991.

7. Xu G, Han T, Yao MH, Xie J, Xu HX, Wu R. Threedimensional ultrasonography for the prediction of breast cancer prognosis. Journal of BUON. 2014; 19:643-649.

8. Wasiuk A, Dalton DK, Schpero WL, Stan RV, ConejoGarcia JR, Noelle RJ. Mast cells impair the development of protective anti-tumor immunity. Cancer immunology, immunotherapy. 2012; 61:2273-2282.
9. Hamilton JP. Epigenetics: principles and practice. Digestive diseases. 2011; 29:130-135.

10. Selaru FM, David S, Meltzer SJ, Hamilton JP. Epigenetic events in gastrointestinal cancer. The American journal of gastroenterology. 2009; 104:1910-1912.

11. Ye M, Du YL, Nie YQ, Zhou ZW, Cao J, Li YF. Overexpression of activated leukocute cell adhesion molecule in gastric cancer is associated with advanced stages and poor prognosis and miR-9 deregulation. Molecular medicine reports. 2015; 11:2004-2012.

12. Herszenyi L, Barabas L, Hritz I, Istvan G, Tulassay Z. Impact of proteolytic enzymes in colorectal cancer development and progression. World journal of gastroenterology. 2014; 20:13246-13257.

13. Tusher VG, Tibshirani R, Chu G. Significance analysis of microarrays applied to the ionizing radiation response. Proceedings of the National Academy of Sciences of the United States of America. 2001; 98:5116-5121.

14. Koboldt DC, Fulton RS, Mclellan MD, Schmidt H, Kalickiveizer J, Mcmichael JF, Fulton L, Dooling DJ, Ding L, Mardis ER, Wlison RK, Ally A, Balasundaram M, et al. Comprehensive molecular portraits of human breast tumours. Nature. 2012; 490:61-70.

15. Gao J, Aksoy BA, Dogrusoz U, Dresdner G, Gross B, Sumer SO, Sun Y, Jacobsen A, Sinha R, Larsson E, Cerami E, Sander C, Schultz N. Integrative analysis of complex cancer genomics and clinical profiles using the cBioPortal. Science signaling. 2013; 6:2383-2383.

16. Murie C, Barette C, Lafanechere L, Nadon R. Control-Plate Regression (CPR) Normalization for High-Throughput Screens with Many Active Features. Journal of biomolecular screening. 2013; 19:661-671.

17. Chen X. Curve-based clustering of time course gene expression data using self-organizing maps. Journal of bioinformatics and computational biology. 2009; 7:645-661.

18. Hong G, Zhang W, Li H, Shen X, Guo Z. Separate enrichment analysis of pathways for up- and downregulated genes. Journal of the Royal Society Interface. 2013; 11: $1154-1158$.

19. Plaisier CL, Pan M, Baliga NS. A miRNA-regulatory network explains how dysregulated miRNAs perturb oncogenic processes across diverse cancers. Genome Research. 2012; 22:2302-2314

20. Hanahan D, Weinberg RA. The hallmarks of cancer.Cell. $2000 ; 100: 57-70$.

21. Hanahan D, Weinberg RA. The hallmarks of cancer: the next generation. Cell. 2011; 144:646-674.

22. Wang E, Zamana N, Mcgeea S, Milanesea JS, MasoudiNejadf A, O'Connor-McCourta M. Predictive genomics: a cancer hallmark network framework for predicting tumor clinical phenotypes using genome sequencing data. Seminars in Cancer Biology. 2015; 30:4-12

23. Mahmood M, Mustafa T, Xu Y, Nima Z, Kannarpady G, Bourdo S, Casciano D, Biris AS. Calcium-channel blocking 
and nanoparticles-based drug delivery for treatment of drug-resistant human cancers. Therapeutic delivery. 2014; 5:763-780.

24. Gielen PR, Aftab Q, Ma N, Chen VC, Hong X, Lozinsky S, Naus CC, Sin WC. Connexin43 confers Temozolomide resistance in human glioma cells by modulating the mitochondrial apoptosis pathway. Neuropharmacology. 2013; 75:539-548.

25. Liao AJ, Fu BB, Wang HH, Li YC, Yao K, Zhang R, Yang W, Liu ZG. Effect of bortezomib on MAPK signaling pathway of K562/DNR cells. Journal of experimental hematology. 2010; 18:1460-1463.

26. Cai W, Rudolph JL, Harrison SM, Jin L, Frantz AL, Harrison DA, Andres DA. An evolutionarily conserved Rit GTPase-p38 MAPK signaling pathway mediates oxidative stress resistance. Molecular biology of the cell. 2011; 22:3231-3241.

27. Sun B, Kawahara M, Nagamune T. Modeling tandem AAG8-MEK inhibition in melanoma cells. Cancer medicine. 2014; 3:710-718.

28. Becerradiaz M, Valderramacarvajal H, Terrazas LI. Signal Transducers and Activators of Transcription (STAT) family members in helminth infections. International journal of biological sciences. 2011; 7:1371-1381.
29. Rovetta AI, Pena D, Hernandez Del Pino RE, Recalde GM, Pellegrini J, Bigi F, Musella RM, Palmero DJ, Gutierrez M, Colombo MI, García VE. IFNG-mediated immune responses enhance autophagy against Mycobacterium tuberculosis antigens in patients with active tuberculosis. Autophagy. 2014; 10:2109-2121.

30. Setta A, Barrow PA, Kaiser P, Jones MA. Immune dynamics following infection of avian macrophages and epithelial cells with typhoidal and non-typhoidal Salmonella enterica serovars; bacterial invasion and persistence, nitric oxide and oxygen production, differential host gene expression, NF-kappaB signalling and cell cytotoxicity. Veterinary immunology and immunopathology. 2012; 146:212-224.

31. Herrera ZM, Ramos TC. Pilot study of a novel combination of two therapeutic vaccines in advanced non-small-cell lung cancer patients. Cancer immunology, immunotherapy. 2014; 63:737-747.

32. Moser EK, Hufford MM, Braciale TJ. Late engagement of CD86 after influenza virus clearance promotes recovery in a FoxP3+ regulatory $\mathrm{T}$ cell dependent manner. PLoS pathogens. 2014; 10:e1004315-e1004315. 